LBL-36250

UC-414

Presented at the NATO Advanced Research Workshop

"Hot Hadronic Matter: Theory and Experiment",

Divonne-les-Bains, France, 27 June - 1 July 1994, and to be published in the Proceedings

The Physics and Experimental Program of the Relativistic Heavy Ion Collider (RHIC)

\author{
John W. Harris \\ Lawrence Berkeley Laboratory \\ University of California, Berkeley, CA 94720
}

September 1994

This work was supported by the Director, Office of Energy Research, Division of Nuclear Physics of the Office of High Energy and Nuclear Physics of the U.S. Department of Energy under Contract DEÁC03-76SF00098 


\section{DISCLAIMER}

This report was prepared as an account of work sponsored by an agency of the United States Government. Neither the United States Government nor any agency thereof, nor any of their employees, make any warranty, express or implied, or assumes any legal liability or responsibility for the accuracy, completeness, or usefulness of any information, apparatus, product, or process disclosed, or represents that its use would not infringe privately owned rights. Reference herein to any specific commercial product, process, or service by trade name, trademark, manufacturer, or otherwise does not necessarily constitute or imply its endorsement, recommendation, or favoring by the United States Government or any agency thereof. The views and opinions of authors expressed herein do not necessarily state or reflect those of the United States Government or any agency thereof. 


\section{DISCLAIMER}

Portions of this document may be illegible in electronic image products. Images are produced from the best available original document. 


\title{
THE PHYSICS AND EXPERIMENTAL PROGRAM OF THE RELATIVISTIC HEAVY ION COLLIDER (RHIC)
}

\author{
John W. Harris* \\ Lawrence Berkeley Laboratory \\ University of California \\ Berkeley, CA 94720 USA
}

\section{INTRODUCTION}

The primary motivation for studying nucleus-nucleus collisions at relativistic and ultrarelativistic energies is to investigate matter at high energy densities ( $\varepsilon \gg 1 \mathrm{GeV} / \mathrm{fm}^{3}$ ). Early speculations of possible exotic states of matter focused on the astrophysical implications of abnormal states of dense nuclear matter. ${ }^{1}$ Field theoretical calculations predicted abnormal nuclear states and excitation of the vacuum. ${ }^{2}$ This generated an initial interest among particle and nuclear physicists to transform the state of the vacuum by using relativistic nucleus-nucleus collisions. ${ }^{3}$ Extremely high temperatures, above the Hagedorn limiting temperature, were expected and a phase transition to a system of deconfined quarks and gluons, the Quark-Gluon Plasma (QGP), ${ }^{4}$ was predicted. Such a phase of matter would have implications for both early cosmology and stellar evolution. The understanding of the behavior of high temperature nuclear matter is still in its early stages. However, the dynamics of the initial stages of these collisions, which involve hard partonparton interactions, can be calculated using perturbative QCD. 5 Various theoretical approaches have resulted in predictions that a high temperature $(\mathrm{T}-500 \mathrm{MeV})$ gluon gas will be formed in the first instants (within $0.3 \mathrm{fm} / \mathrm{c}$ ) of the collision. ${ }^{6}$ Furthermore, QCD lattice calculations ${ }^{7}$ exhibit a phase transition between a QGP and hadronic matter at a temperature near $250 \mathrm{MeV}$. Such phases of matter may have existed shortly after the Big Bang and may exist in the cores of dense stars. An important question is whether such states of matter can be created and studied in the laboratory. The Relativistic Heavy Ion Collider (RHIC) ${ }^{8}$ and a full complement of detector systems are being constructed at Brookhaven National Laboratory to investigate these new and fundamental properties of matter. 9

${ }^{*}$ Alexander von Humboldt Foundation U.S. Senior Scientist Award Recipient visiting the Institute für Kernphysik, Universität Frankfurt, Frankfurt, Germany. 


\section{THE RELATIVISTIC HEAVY ION COLLIDER (RHIC)}

In 1983 the U.S. Nuclear Science Advisory Committee (NSAC) Long Range Plan 10 stated that the construction of RHIC was the highest priority new scientific opportunity in the field. This high priority for RHIC was reaffirmed in subsequent NSAC Meetings and by the National Research Council. 11 The U.S. High Energy Physics Panel (HEPAP) stated 12 in 1985 that "although (RHIC) is in the realm of nuclear physics, it is of interest to the particle physics community. It will be directly testing non-perturbative aspects of the non-Abelian gauge theory of quarks and gluons." RHIC was proposed for construction at Brookhaven National Laboratory and construction was started in January 1991. RHIC is expected to begin operation for physics in March 1999.

A schematic diagram of the RHIC accelerator complex at Brookhaven is displayed in Fig. 1. Nuclear beams are accelerated from the tandem Van de Graaff accelerator through a transfer line into the AGS Booster synchrotron and then into the AGS, which serves as an injector for RHIC. RHIC will accelerate and collide ions from protons up to the heaviest nuclei over a range of energies, up to $250 \mathrm{GeV}$ for protons and $100 \mathrm{GeV} /$ nucleon for $\mathrm{Au}$ nuclei. Fig. 2 summarizes the capabilities of the accelerator. In addition to the colliding beams described in Fig. 2, plans are underway to inject and accelerate polarized protons at RHIC in order to study the spin content of the proton. ${ }^{13}$

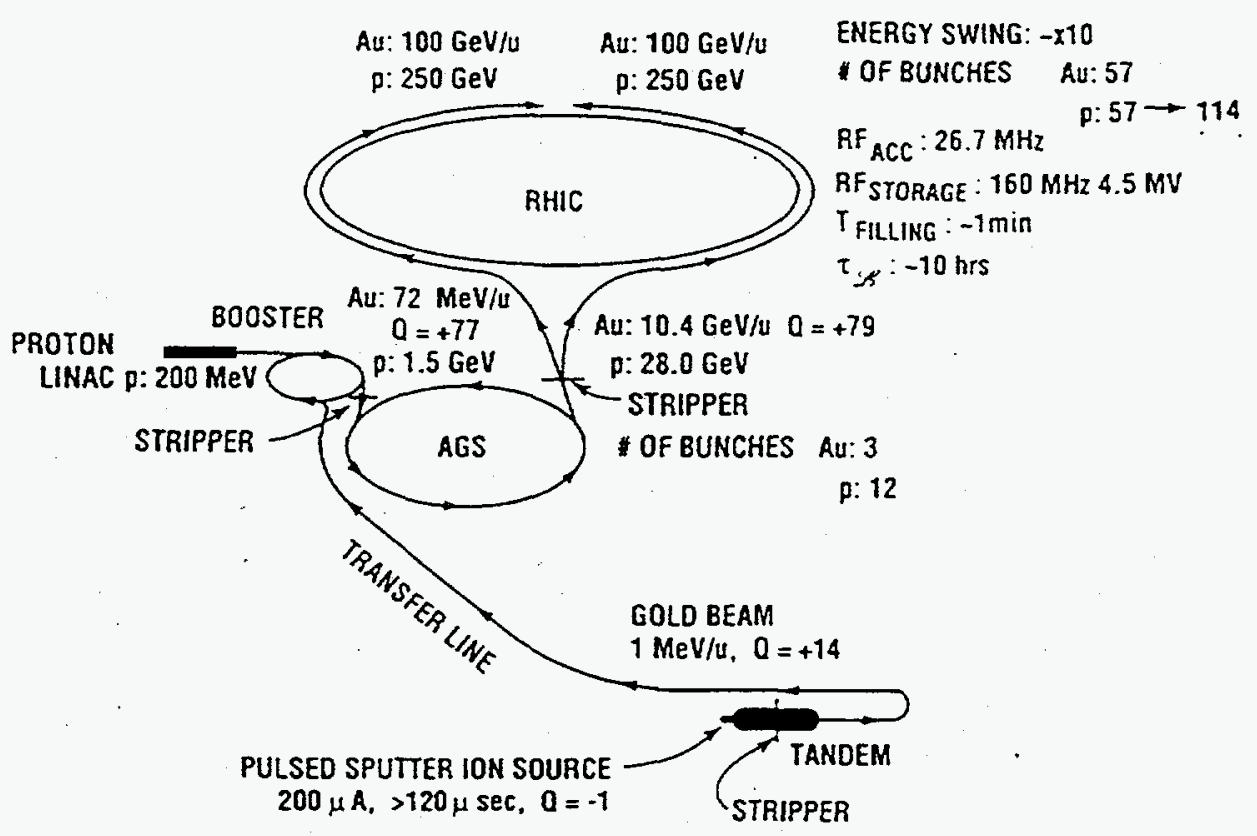

Figure 1. The Relativistic Heavy Ion Collider (RHIC) accelerator complex at Brookhaven National Laboratory. Nuclear beams are accelerated from the tandem Van de Graaff, through the transfer line into the AGS Booster and AGS prior to injection into RHIC. Details of the characteristics of proton and Au beams are also indicated after acceleration in each phase. 


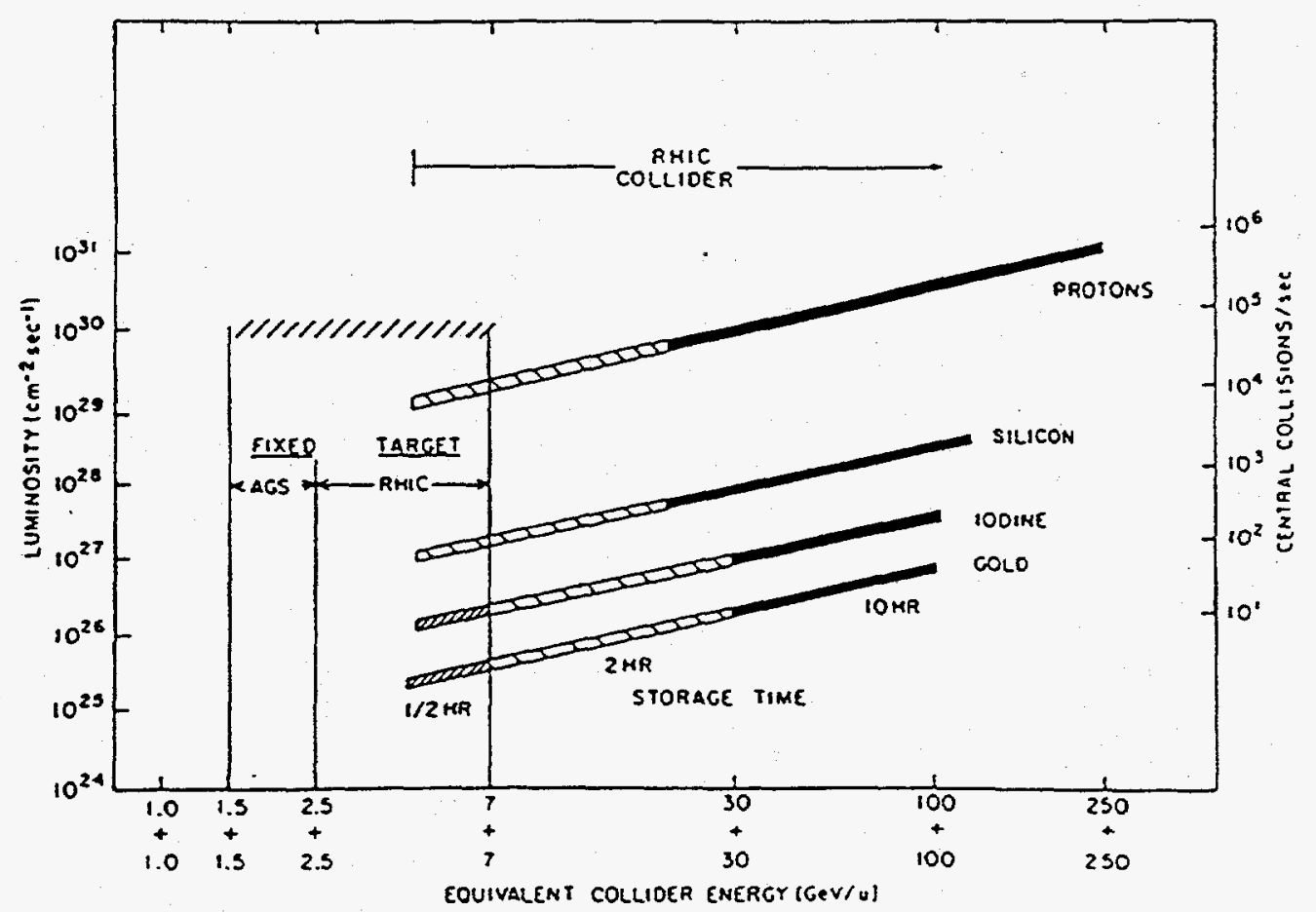

Figure 2. The RHIC luminosity and number of central collisions per second, for impact parameters less than 1 Fermi, are plotted as a function of the colliding beam energies for various projectile systems.

\section{RHIC EXPERIMENTS}

Collisions of the heaviest nuclei at impact parameters near zero at RHIC are expected to produce approximately 1000 charged particles per unit pseudorapidity. ${ }^{14}$ This presents a formidable environment in which to detect the products of these reactions. The experiments will take various different approaches to search for the QGP. Two large collider detectors have full scientific, technical, cost and schedule approval for construction at RHIC. These are the STAR and PHENIX detector systems, both of which have begun construction and anticipate operation at RHIC start-up. The STAR experiment will concentrate on measurements of hadron production over a large solid angle in order to study global observables on an event-by-event basis. The PHENIX experiment will concentrate on measurements of lepton and photon production and will have the capability of measuring hadrons in a limited range of pseudorapidity. In addition, two smaller experiments have scientific approval and are in the technical development stage. These are a forward and midrapidity hadron spectrometer experiment (BRAHMS) and a compact multiparticle spectrometer (PHOBOS). The collaborations, which intend to construct these detector systems and exploit their capabilities, consist of more than 600 scientists from 80 institutions and 15 countries. A brief description of each experiment and its physics goals will be presented.

\section{The STAR Experiment}

The STAR (Solenoidal Tracker At RHIC) experiment ${ }^{15}$ will search for signatures of QGP formation and investigate the behavior of strongly interacting matter at high energy density. A flexible detection system will be utilized to simultaneously measure many experimental observables to study signatures of the QGP phase transition as well as the space-time evolution of the collision process. Measurements will be made at midrapidity 
over a large pseudorapidity range $(|\eta|<2)$ with full azimuthal coverage $(\Delta \phi=2 \pi)$ and azimuthal symmetry. The detection system is shown in Fig. 3. It will consist of a silicon vertex tracker (SVT) and time projection chamber (TPC) inside a solenoid magnet with a $0.5 \mathrm{~T}$ uniform field for tracking, momentum analysis and particle identification via $\mathrm{dE} / \mathrm{dx}$; a time-of-flight system (TOF) surrounding the TPC to extend particle identification to higher momenta; electromagnetic calorimetry (EMC) directly inside the solenoid coil to measure the transverse energy of events and to trigger on and measure high transverse momentum particles and jets; and external time projection chambers (XTPC) to extend the STAR tracking coverage to forward pseudorapidities. The following trigger detectors are also under construction for implementation in STAR (see Fig. 3): a central trigger barrel (CTB), TPC endcap trigger (not shown), vertex position detector (VPD) and the forward veto calorimeters (at large pseudorapidities, not shown).

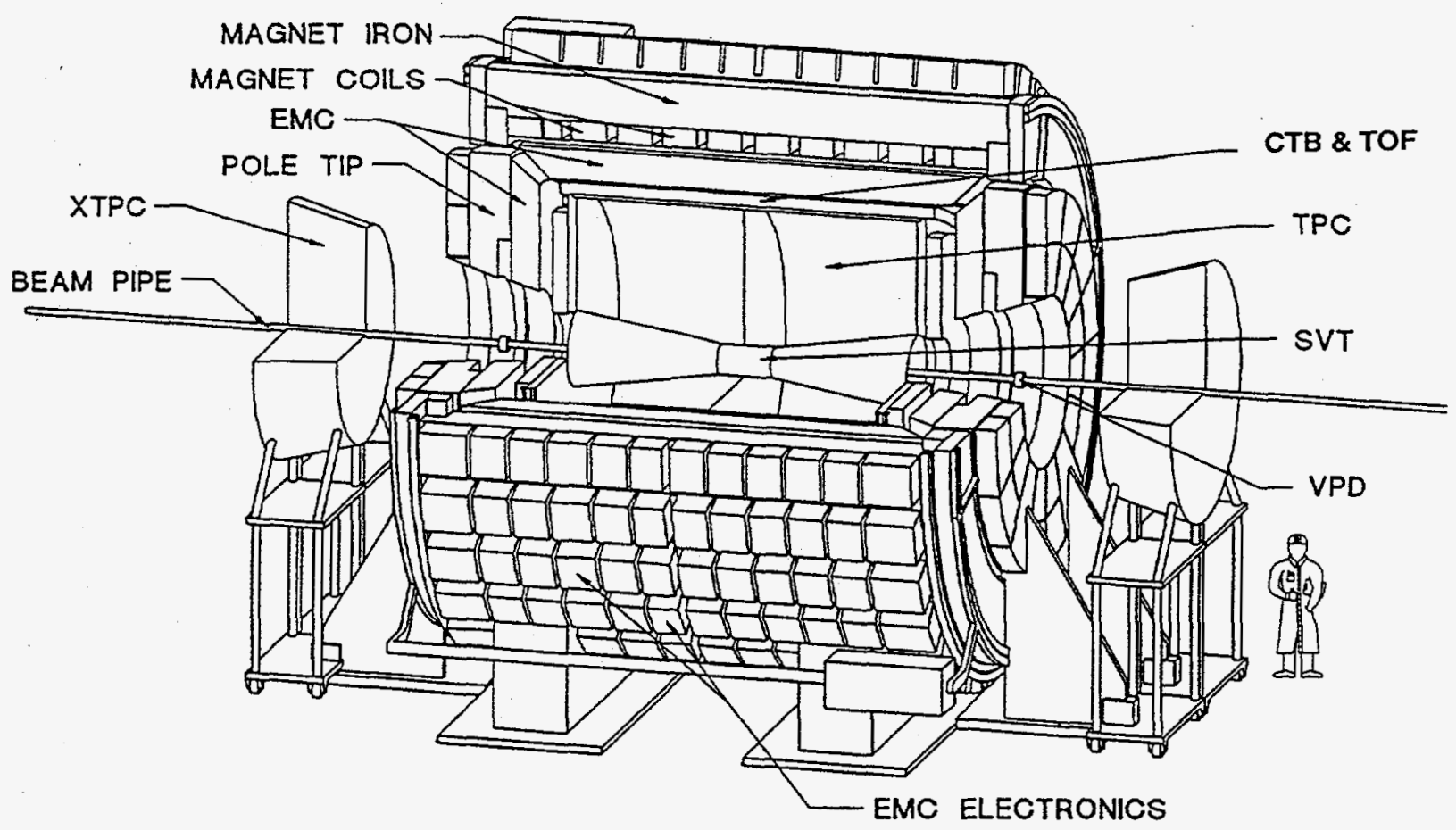

Figure 3. Layout of the STAR experiment at RHIC.

STAR will exploit two aspects of hadron production that are fundamentally new at RHIC. One of these is the measurement of global observables on an event-by-event basis. The event-by-event measurements of global observables possible using STAR are:

- Temperature of $\pi^{ \pm},\left\langle\mathrm{p}_{\mathrm{t}}>\right.$ of $\pi^{ \pm}$and $\mathrm{K}^{ \pm}$

- Flavor content (strangeness using $\mathrm{K}^{ \pm}$multiplicity, $\mathrm{K} / \pi$ ratio)

- Charged-particle multiplicity

- Entropy density

- Pion source size

- Reaction dynamics and collision geometry

- Neutral transverse and total transverse energy

- Degree of fluctuations in

$\mathrm{d}^{3} \mathrm{n} / \mathrm{dp} \mathrm{p}_{t} \mathrm{~d} \eta \mathrm{d} \phi$

Energy $\left(d^{2} E_{t} / d \eta d \phi\right)$

Entropy

Isospin (neutral/charged particle energy) 
- Stopping \& forward baryon distributions

- Particle ratios

The event-by-event measurement of these global observables is possible because of the very high charged particle densities, $\mathrm{dn}_{\mathrm{ch}} / \mathrm{d} \eta \approx 1000$ expected in nucleus-nucleus collisions at RHIC. This will allow novel determination of the thermodynamic properties of single events. For example, the slope of the transverse momentum $\left(\mathrm{p}_{\mathrm{t}}\right)$ distribution for pions and the $\left\langle\mathrm{p}_{t}\right\rangle$ for pions and kaons can be determined event-by-event. Individual events can be characterized by a "temperature" to search for events with extremely high temperature, predicted ${ }^{16}$ to result from deflagration of a QGP. Displayed in Fig. 4 are two spectra generated by the Monte Carlo method from Maxwell-Boltzmann distributions with $T=150$ and $250 \mathrm{MeV}$, each containing 1000 pions. The slopes of spectra with $\mathrm{T}=150$ and $250 \mathrm{MeV}$ derived from fits using a Maxwell-Boltzmann distribution, also shown in Fig. 4, can easily be discriminated at the single event level. The determination of $\left\langle p_{t}\right\rangle$ for pions can be made very accurately on the single event basis in this experiment, over the expected range of multiplicities in central collisions from $\mathrm{Ca}+\mathrm{Ca}$ to $\mathrm{Au}+\mathrm{Au}$. The $\left\langle\mathrm{p}_{\mathrm{t}}\right\rangle$ of kaons $\mathrm{can}$ be determined accurately for single events from central $\mathrm{Au}+\mathrm{Au}$ events.

Correlations between such observables will be made on an event-by-event basis to isolate potentially interesting event types for detailed investigation. Events with special (often extreme) values of many of the above distributions can be available at the trigger level to be able to enhance the sensitivity of STAR, increase statistics for specialized events and increase the likelihood of new discoveries. The full azimuthal coverage, particle identification and continuous tracking of STAR is required to perform these measurements.

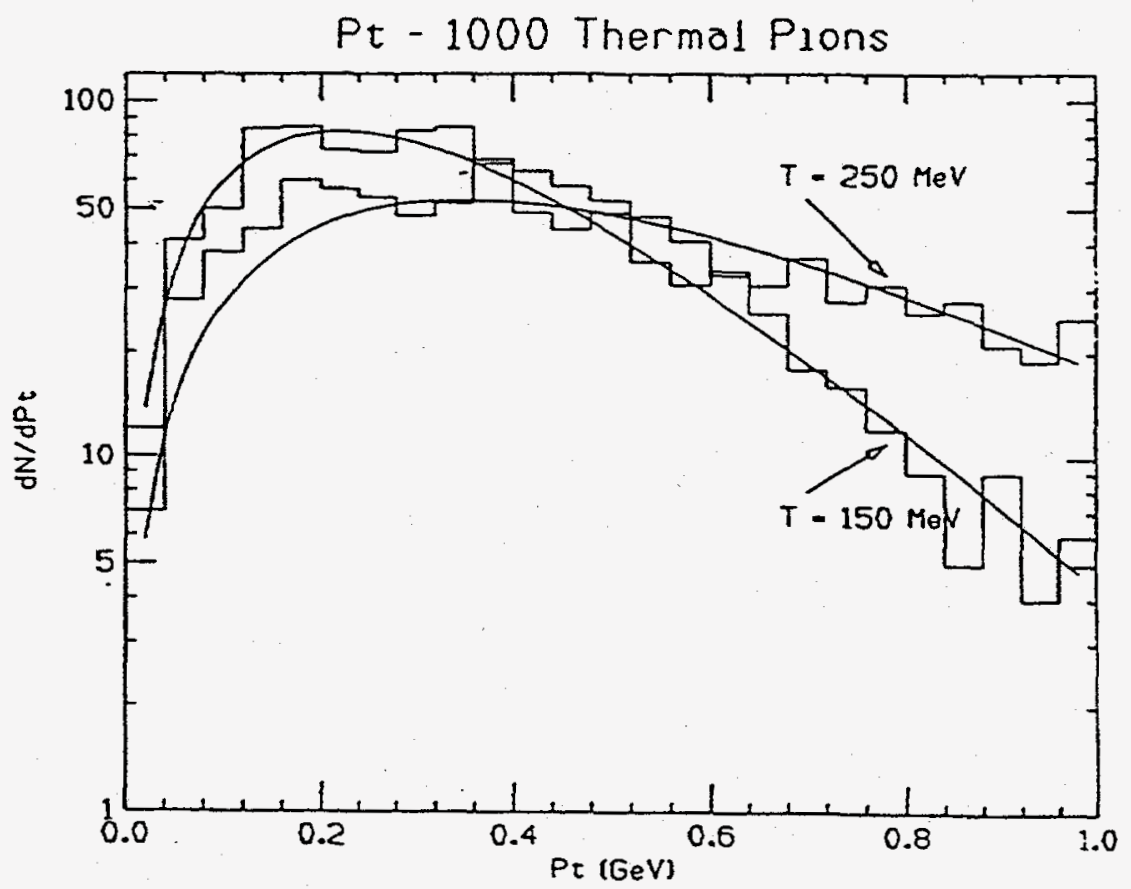

Figure 4. Simulation of the $\mathrm{p}_{t}$ spectrum for one event generated using a Boltzmann distribution of 1000 pions. The histograms correspond to single events generated with $T=150 \mathrm{MeV}$ and $250 \mathrm{MeV}$. The curves are fits to the histogram using a Maxwell-Boltzmann distribution. 
In addition to the event-by-event observables, inclusive measurements will be performed in STAR and then correlated on an ensemble basis. From among these inclusive measurements, those of particular interest at RHIC are measurements of $\phi$-mesons, shortlived singly-strange hadrons $\left(\Lambda, \bar{\Lambda}, \mathrm{K}_{\mathrm{s}}^{\mathrm{s}}\right)$, multiply-strange baryons $\left(\Xi^{ \pm}, \Omega^{-}\right)$, and $\mathrm{K}_{\mathrm{s}} \mathrm{K}^{\circ}{ }_{\mathrm{s}}$ particle interferometry. The production cross section, mass and width of $\phi$-mesons can be measured in STAR from the decay $\phi \Rightarrow \mathrm{K}^{+}+\mathrm{K}^{-}$. The $\phi$ production rate, mass and width are expected to be extremely sensitive to changes in the quark masses ${ }^{17,18,19}$ due to a chiral phase transition at high energy densities. Enhancements to the strange antibaryon content due to QGP formation have been predicted. ${ }^{20}$ Furthermore, multiply-strange baryons ( $\Xi \pm$, $\Omega^{-}$) are expected to be more sensitive to the existence of the QGP than singly-strange hadrons. ${ }^{21}$ Examples of the mass spectra expected for $\Lambda$ and $\Xi-$ in STAR are shown in Fig. 5. The $\mathrm{K}_{\mathrm{s}}^{\mathrm{O}} \mathrm{K}^{\mathrm{o}}{ }_{\mathrm{s}}$ correlations will allow two-particle Bose-Einstein measurements without Coulomb distortions.

The other aspect of hadron production that is fundamentally new at RHIC is the use of hard scattering of partons as a probe of the properties of high density nuclear matter. Measurable yields of intermediate and high pt particles and high $\mathrm{p}_{\mathrm{t}}$ jets at RHIC will allow investigations of hard QCD processes via both segmented calorimetry and high $\mathrm{p}_{\mathrm{t}}$ single particle measurements. Measurements of the remnants of hard-scattered partons will be used as a penetrating probe of the QGP, will provide important new information on gluon distributions in nucleons and gluon shadowing in nuclei, and provide measurements to test perturbative QCD in polarized proton (spin) studies.
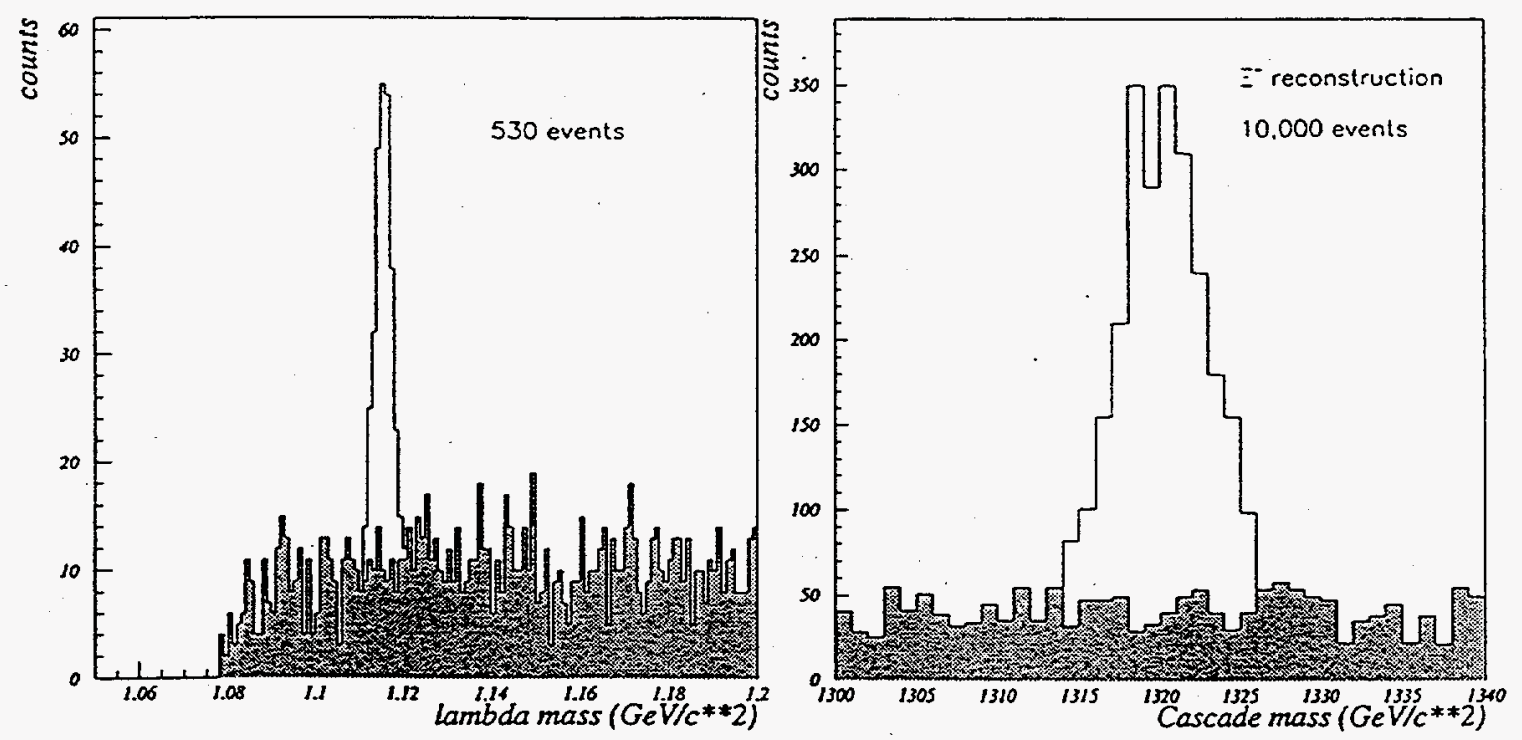

Figure 5. Simulated invariant mass spectra for $\Lambda$ and $\Xi^{-}$measured in STAR using the SVT and TPC. 
The physics goals of PHENIX (Pioneering High Energy Nuclear Interaction eXperiment) ${ }^{22}$ are to measure as many potential signatures of the QGP as possible as a function of a well-defined common variable such as impact parameter or pseudorapidity density. PHENIX will measure lepton pairs (di-electrons and di-muons), photons and hadrons. The experiment will be sensitive to very small cross section processes such as the production of the $\mathrm{J} / \psi, \psi^{\prime}, \Upsilon$ and high pt spectra. It will also have the capability for high rates with $p p$ and $\mathrm{pA}$ collisions.

A diagram of the PHENIX detector system is displayed in Fig. 6. The magnet has an axial field along the beam direction with tracking chambers and detectors for the identification of electrons, muons, photons and hadrons installed outside the field. There are two arms for dielectron measurements, each with 1 steradian acceptance at midrapidity. Each arm is equipped with a ring-imaging Cerenkov detector (RICH), a time-expansion chamber (TEC) for $\mathrm{dE} / \mathrm{dx}$ measurements, time-of-flight detectors (TOF), and an electromagnetic calorimeter (EM Cal). Photons and hadrons will also be measured at midrapidity with 2 and 0.36 steradian acceptances, respectively. A separate muon spectrometer with 1 steradian acceptance is located at forward rapidities as shown in Fig. 6. The magnetic field in the muon arm is transverse to the beam direction. A silicon multiplicity-vertex detector (MVD) and beam-beam coincidence counters are located near the interaction vertex. The MVD covers $|\eta|<2.7$ about midrapidity for event selection via charged-particle multiplicity.

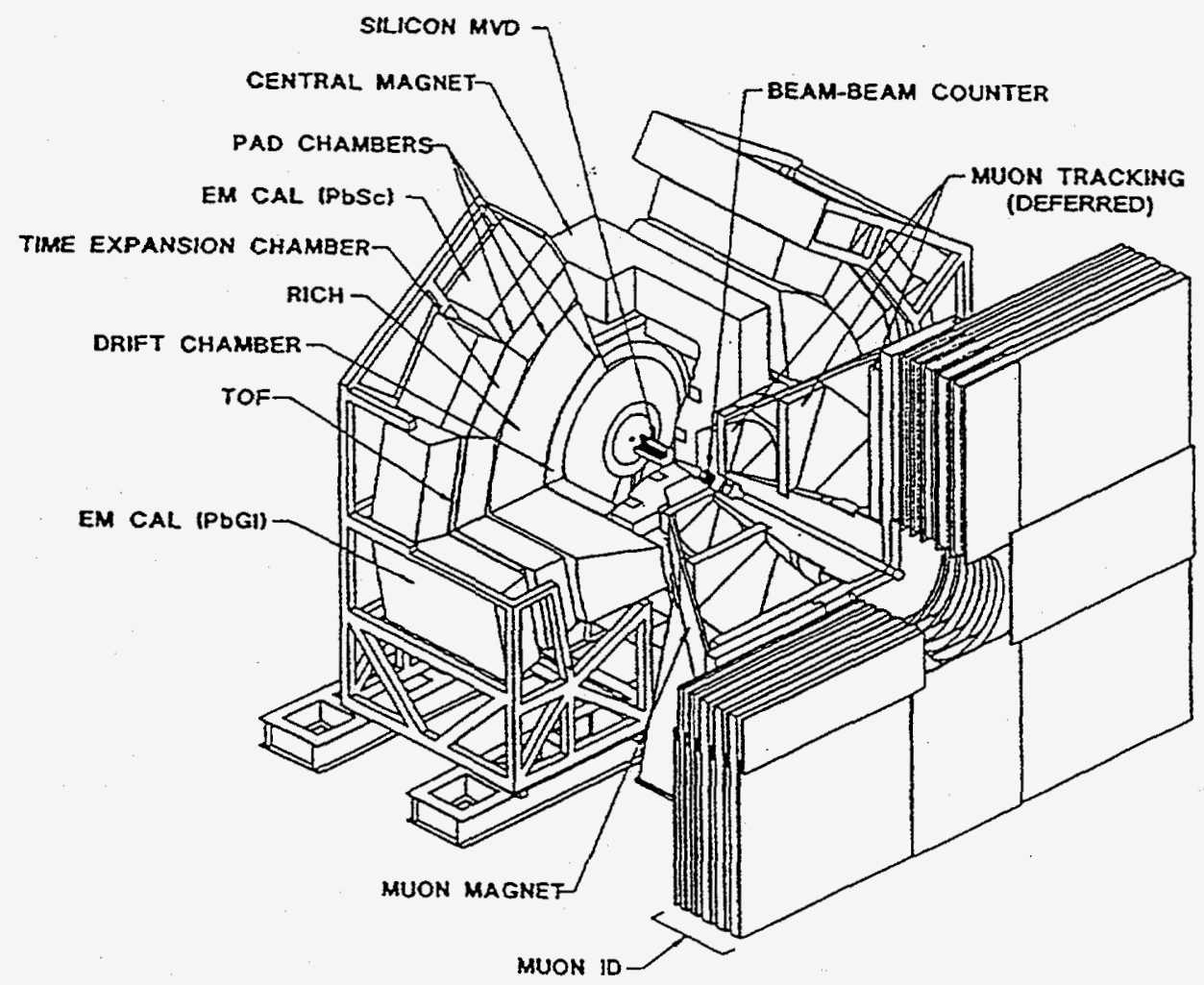

Figure 6. A diagram of the PHENIX experiment at RHIC. The beams collide along the horizontal direction in the center of the detector. The detector components are defined in the text.

PHENIX will investigate the degree of deconfinement in RHIC collisions, and thus the possibility of QGP formation, by measuring the yields of $J / \psi, \psi^{\prime}, \Upsilon$. If the $J / \psi$ radius is larger than the Debye screening length then the QCD potential between the cc pairs, which 
form a $J / \psi$, is weakened and $J / \psi$ formation suppressed. ${ }^{23}$ Since the $J / \psi, \psi^{\prime}, \Upsilon$ radii are different, i.e. $r\left(\psi^{\prime}\right)>r(J / \psi)>r(\Upsilon)$, a study of the relative suppression of each of these is sensitive to the screening in a QGP and will help differentiate between deconfinement and possible dissociation in hot nuclear matter. PHENIX will measure $\mathrm{J} / \psi$ via electron-pairs near midrapidity and $\mathrm{J} / \psi, \psi^{\prime}, \mathrm{T}$ via muon-pairs at forward angles.

The anticipated raw muon-pair mass spectrum in the forward muon arm is displayed in Fig. 7 for one-month of RHIC operation at the design luminosity for central $\mathrm{Au}+\mathrm{Au}$ collisions. Peaks for the $J / \psi, \psi^{\prime}, \Upsilon$ can be observed. With subtraction of like-sign muonpairs, and application of kinematic cuts the peak-to-background of this spectrum is expected to improve further and the $\psi^{\prime}$ peak will become more prominent. For one year of running at the RHIC design luminosity of $2 \times 10^{26} \mathrm{~cm}^{-2} \mathrm{~s}^{-1}$, PHENIX will detect $390 \mathrm{~K}$ $\mathrm{J} / \psi, 5.7 \mathrm{~K} \psi^{\prime}$ and $1.2 \mathrm{~K} \Upsilon$ for central collisions (top $10 \%$ multiplicity) of Au + Au. The mass resolution is about $100 \mathrm{MeV}$ for the $\mathrm{J} / \psi$ and the pion rejection is somewhat better than $10^{-4}$ at $\mathrm{p} \geq 2.5 \mathrm{GeV} / \mathrm{c}$. In addition to these charmonium studies, PHENIX will measure open charm (D $\bar{D}$ decays) via unlike-sign $\mathrm{e} \mu$ coincidences using the electron and muon spectrometers. The rate of open charm production is expected to be sensitive to details of the early stages of RHIC collisions and will provide complimentary information to the charmonium studies.

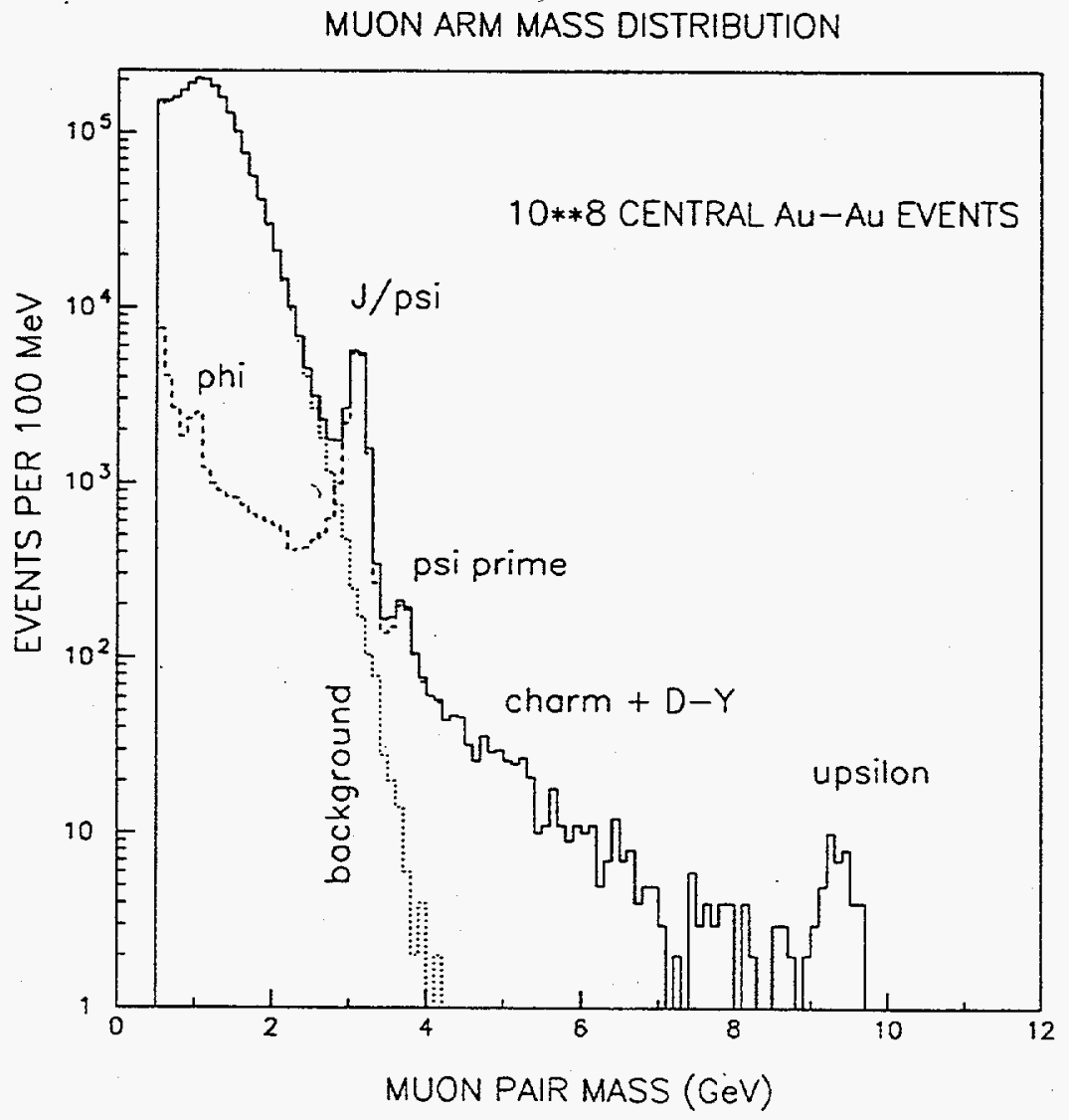

Figure 7. PHENIX muon-pair mass spectrum anticipated in one month of RHIC nunning for central $\mathrm{Au}+\mathrm{Au}$ collisions at a luminosity of $2 \times 10^{26} \mathrm{~cm}^{-2} \mathrm{~s}^{-1}$.

PHENIX will investigate possible chiral symmetry restoration by performing high resolution measurements of the leptonic and hadronic decays of $\phi$-mesons. In a chirallyrestored QGP both the $\phi$-meson and kaon masses may be modified resulting in change in the mass and width of the $\phi$-meson and the branching ratio between leptonic and hadronic channels. ${ }^{24}$ The anticipated electron-pair spectrum for 8 days of central trigger $\mathrm{Au}+\mathrm{Au}$ 
operation at RHIC is shown in Fig. 8. Extremely sharp peaks for the $\omega, \phi$ and $J / \psi$ are observed, with a 2-to-1 signal-to-noise ratio in the low mass resonance region. The mass resolution is approximately $4 \mathrm{MeV}$ for the $\phi \rightarrow \mathrm{e}^{+} \mathrm{e}^{-}$(and $<1 \mathrm{MeV}$ for $\phi \rightarrow \mathrm{K}^{+} \mathrm{K}^{-}$in the hadronic measurement). The pion rejection is better than $10^{-4}$ for $\mathrm{p} \leq 4 \mathrm{GeV} / \mathrm{c}$.

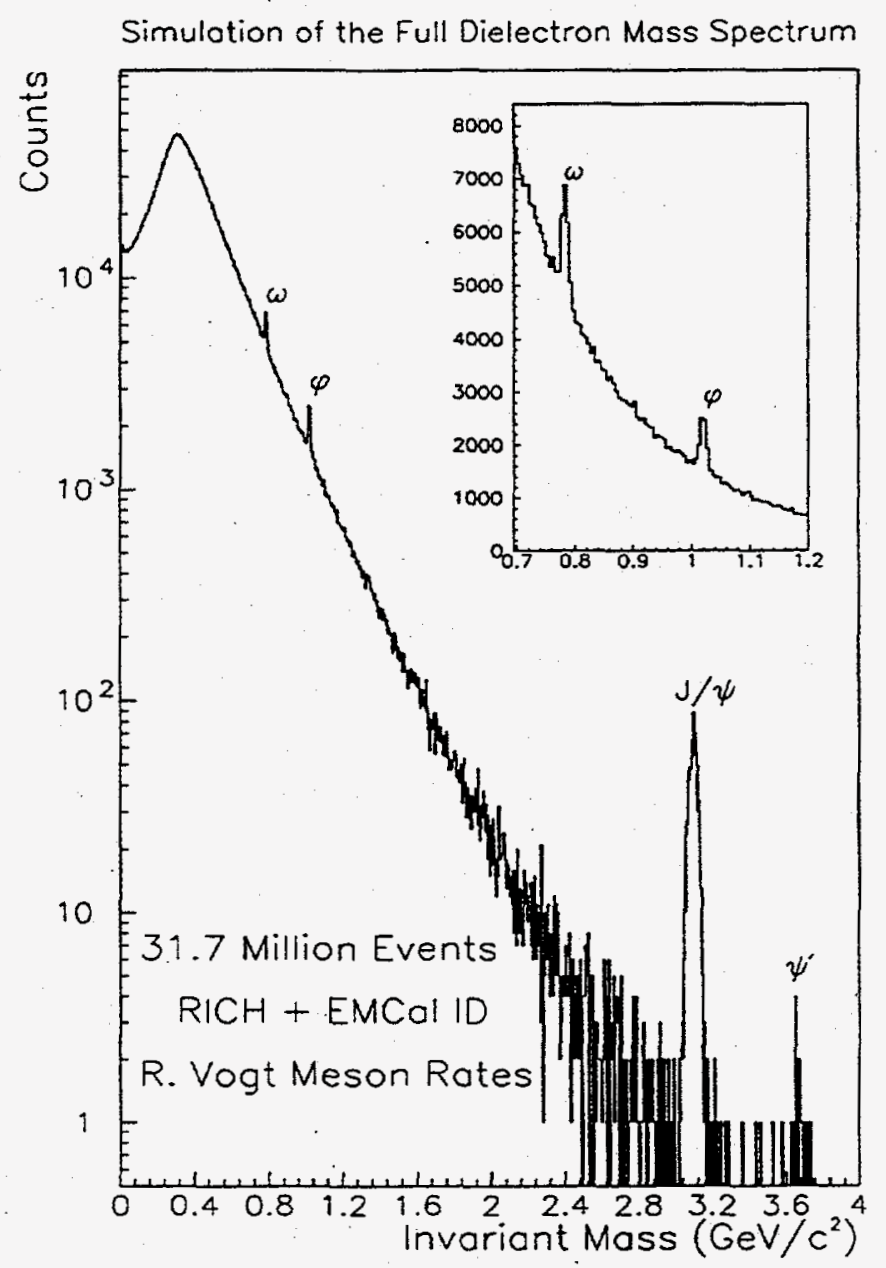

Figure 8. PHENIX electron-pair mass spectrum anticipated in 8 days of RHIC running for central $\mathrm{Au}+\mathrm{Au}$ collisions at a luminosity of $2 \times 10^{26} \mathrm{~cm}^{-2} \mathrm{~s}^{-1}$.

There are other measurements of interest that will be undertaken by PHENIX. Measurements will be made using the electromagnetic calorimeter and high resolution photon detector to determine the yield of direct photons (for $\mathrm{p}_{\mathrm{t}} \geq 0.5 \mathrm{GeV} / \mathrm{c}$ ) radiated from the nuclear interaction. An enhancement of photons with $\mathrm{pt} \geq 2$ to $3 \mathrm{GeV} / \mathrm{c}$ is expected because of the high gluon concentration in the early high energy-density stage of RHIC collisions. ${ }^{25}$ PHENIX will also investigate the order of the QGP phase transition by measuring the $\langle\mathrm{pt}\rangle$ of identified-charged pions, kaons and protons at mid-rapidity as a function of the energy density. By detecting both hadrons and photons, PHENIX can measure fluctuations in isospin, $\pi^{\%} /\left(\pi^{+}+\pi\right)$, which might occur in a second order phase transition. ${ }^{26}$ PHENIX will also study strangeness and charm production for enhancements, jet-quenching, and the space-time evolution of the system as a function of energy density. 
The physics goals of the BRAHMS (BRoad RAnge Hadron Magnetic Spectrometers) experiment ${ }^{27}$ are to achieve a basic understanding of relativistic heavy ion collisions at RHIC through a systematic study of particle production in AA collisions from the peripheral to the most central in impact parameter. Measurements will be performed using two high resolution magnetic spectrometers at various angles to cover both the baryon-rich fragmentation regions and the high temperature, baryon-depleted midrapidity region. A diagram of the BRAHMS forward and midrapidity spectrometers is shown in Fig. 9. The spectrometers will measure and identify inclusive and semi-inclusive $\pi \pm, \mathrm{K}^{ \pm}$and $\mathrm{p}^{ \pm}$, and their momenta with high statistics over a small solid angle and over a wide range of pseudorapidity $(0 \leq \eta \leq 4)$ and transverse momentum. Particle identification is performed using various combinations of time-of-flight arrays, and threshold and ring-imaging Cherenkov counters. BRAHMS will measure inclusive and semi-inclusive particle spectra and extract the net baryon densities temperatures from spectral slopes as a function of rapidity to determine whether thermal and chemical equilibrium are reached in these collisions. It will also be able to study both high and low transverse momentum processes. Centrality will be measured using a global multiplicity detector.

Forward spectrometer

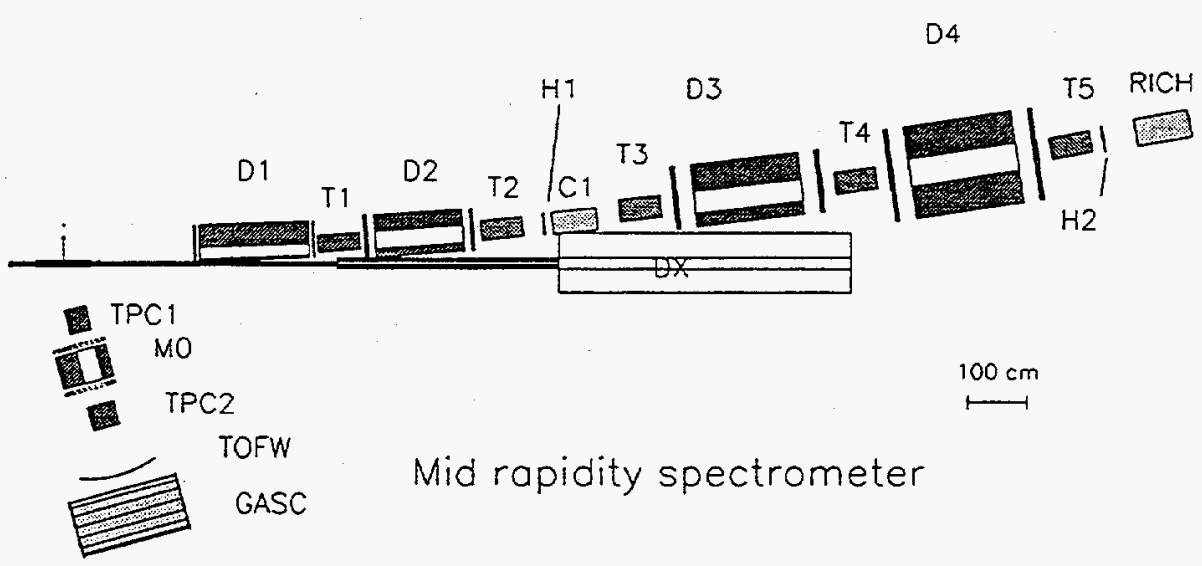

Figure 9. A diagram of the BRAHMS forward and midrapidity spectrometers in the narrow angle hall at RHIC.

\section{The PHOBOS Experiment}

The physics goals of the PHOBOS experiment 28 are to measure single particle spectra and correlations between particles with low transverse momenta and to characterize events using a multiplicity detector. Charged particles will be measured and identified in the range $0 \leq \mathrm{y} \leq 1.5$ and $15 \mathrm{MeV} / \mathrm{c} \leq \mathrm{pt}_{\mathrm{t}} \leq 600 \mathrm{MeV} / \mathrm{c}$ for pions and $45 \mathrm{MeV} / \mathrm{c} \leq \mathrm{pt}_{\mathrm{t}} \leq 1200 \mathrm{MeV} / \mathrm{c}$ for protons. The range of particles to be studied include $\gamma, \pi^{ \pm}, K^{ \pm}, p, \bar{p}, \phi, \Lambda, \bar{\Lambda}, d$ and $\bar{d}$. Particle ratios, $\mathrm{pt}_{\mathrm{t}}$ spectra, strangeness production $\left(\mathrm{K}^{ \pm}, \phi, \Lambda, \bar{\Lambda}\right)$ and particle correlations will be studied. An illustration of the experiment is shown in Fig. 10. The top coils and magnet iron are not shown in order to see the other components. The multiplicity detector consists of silicon strip detectors and silicon pad detectors. The two magnetic arms have a field strength of 2 Tesla. Eleven layers of silicon, 5 layers of pads and 6 layers of strips, are installed in each magnetic spectrometer for tracking and momentum measurements. 


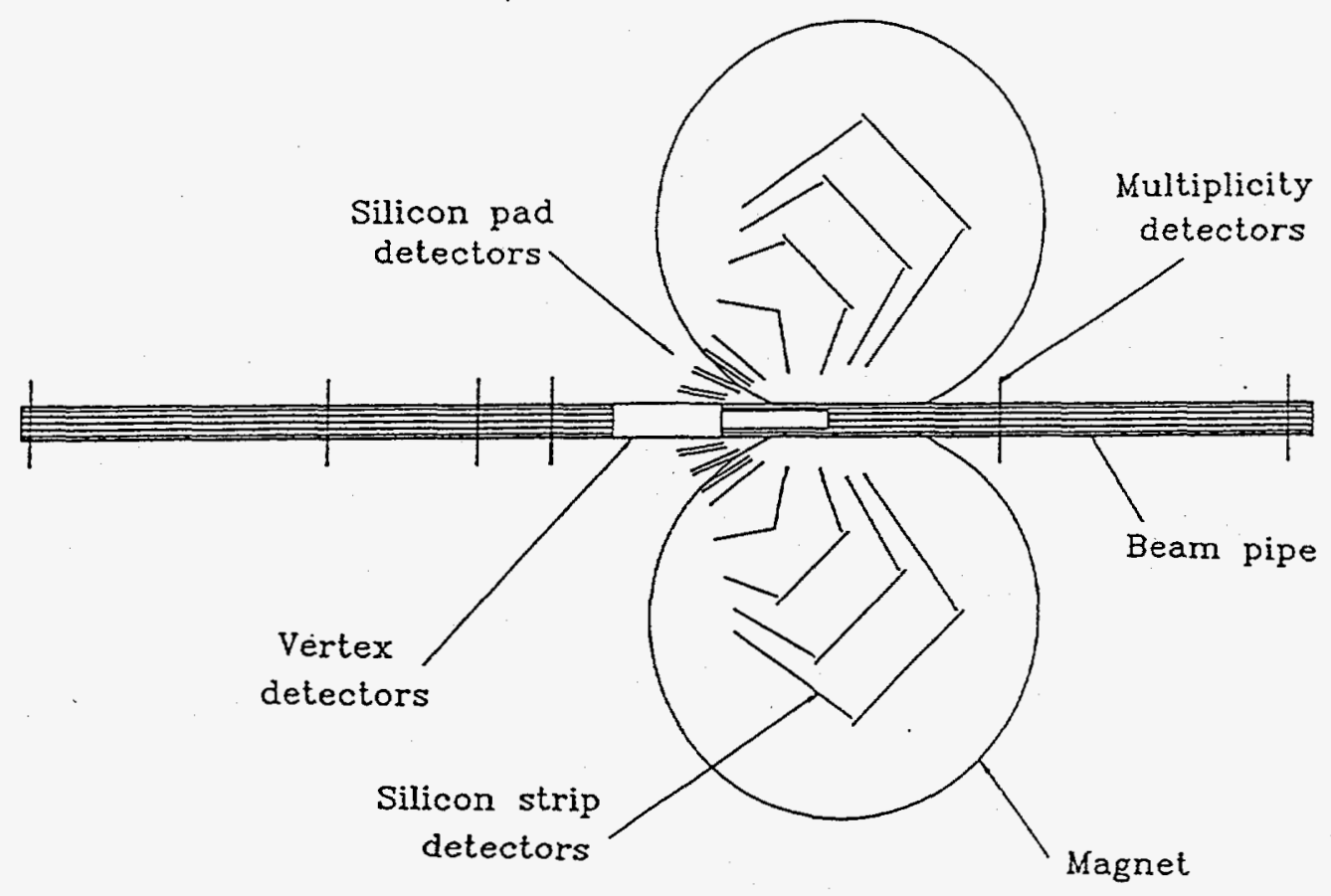

Figure 10. A diagram of the PHOBOS two-arm multiparticle spectrometer. The two arms are located on opposite sides of the beam pipe. Each arm has a 2 Tesla magnet represented by only the lower coils for ease of viewing other parts of the set-up, with silicon detector planes for tracking.

\section{ACKNOWLEDGMENTS}

I would like to thank Timothy Hallman for helpful comments and Joy Lofdahl for assistance with the manuscript. I thank my collaborators in STAR and friends in the PHENIX, BRAHMS, AND PHOBOS Collaborations for their work presented in this manuscript. This work was supported in part by the Alexander von Humboldt Foundation and the Director, Office of Energy Research, Division of Nuclear Physics of the Office of High Energy and Nuclear Physics of the U.S. Department of Energy under contract DEAC03-76SF00098.

\section{REFERENCES}

1. E. Feenberg and H. Primakoff, Phys. Rev. 70, 980 (1946); A.R. Bodmer, Phys. Rev. D4, 1601 (1971);

G. Baym and S.A. Chin, Phys. Lett. 62B, 241 (1976).

2. T.D. Lee and G.C. Wick, Phys. Rev. D9, 2291 (1974).

3. T.D. Lee, Rev. Mod. Phys. 47, 267 (1975).

4. J.C. Collins and MJ. Perry, Phys. Rev. Lett. 34, 1353 (1975); G. Chapline and M. Nauenberg, Phys. Rev.

D16, 450 (1977); L. Susskind, Phys. Rev. D20, 2610 (1979).

5. K. Geiger and B. Mueller, Nucl. Phys. B369, 600 (1992).

6. See presentation of $\mathrm{K}$. Geiger, Proceedings of this Workshop. 
7. J. Kapusta, Nucl. Phys. 61, 461 (1980); J. Kuti et al., Phys. Lett. 95B, 75 (1980); H. Satz, Ann. Rev. Nucl. Part. Sci. 35, 245 (1985).

8. Conceptual Design of the Relativistic Heavy Ion Collider, Brookhaven National Laboratory Report BNL 52195 (1989).

9. For future perspectives utilizing heavy nuclear beams in the Large Hadron Collider, please see the presentation of H.Gutbrod, Proceedings of this Workshop.

10. "A Long Range Plan for Nuclear Science," Report of the DOE/NSF Nuclear Science Advisory Committee, December 1983.

11. "Physics through the 1990s," Panel on Nuclear Physics of the Physics Survey Committee and the National Research Council, National Academy Press, Washington, D.C., 1986.

12. U.S. High Energy Physics Panel (HEPAP) Report, 1985.

13. Proposal on Spin Physics Using the RHIC Polarized Collider, RHIC Spin Collaboration (1992).

14. F. Videbaek and T. Throwe, Fourth Workshop on Experiments and Detectors for a Relativistic Heavy Ion Collider, Brookhaven National Laboratory Report BNL-52262 (1990).

15. Conceptual Design Report for the Solenoidal Tracker At RHIC, STAR Collaboration, PUB-5347 (1992).

16. E.V. Shuryak and O.V. Zhirov, Phys. Lett. B89, 253 (1980); E.V. Shuryak and O.V. Zhirov, Phys. Lett. B171, 99 (1986).

17. R. D. Pisarski and F. Wilczek, Phys. Rev. D29, 338 (1984).

18. T. Hatsuda and T. Kunihiro, Phys. Lett. B185, 304 (1987).

19. E.V. Shuryak, Nucl. Phys. A525, 3c (1991).

20. J. Rafelski and A. Schnabel, "Intersections Between Nuclear and Particle Physics," AIP Proceedings No. 176,1068 (1988), and references therein.

21. J. Rafelski, Phys. Rep. 88, 331 (1982).

22. PHENIX Experiment at RHIC - Conceptual Design Report, PHENIX Collaboration Report (1993).

23. T. Matsui and H. Satz, Phys. Lett. B178 (1986) 416.

24. D. Lissauer and E.V. Shuryak, Phys. Lett. B253 (1991) 15.

25. E. Shuryak and L. Xiong, Phys. Rev. Lett. 70 (1993) 2241; K.J. Eskola and M. Gyulassy, Phys. Rev. C47 (1993) 2239.

26. F. Wilczek, Nuc. Phys. A566 (1994) 123c.

27. Interim Design Report for the BRAHMS Experiment at RHIC, BNL Report, 1994.

28. Conceptual Design Report, PHOBOS Collaboration (1994). 